\title{
Experimental Examination of the Motion of a Tethered System with Large Deformation and Large Displacement ${ }^{*}$
}

\author{
Shoichiro TAKEHARA ${ }^{* *}$ Masahiro NOHMI ${ }^{* * *}$ Yoshiaki TERUMICHI ${ }^{* * *}$ and \\ Kiyoshi SOGABE ${ }^{* * * *}$ \\ **Institute of Industrial Science, The University of Tokyo, \\ 4-6-1 Komaba, Meguro-ku, Tokyo, 153-8505 Japan \\ E-mail: stakeha@iis.u-tokyo.ac.jp \\ ${ }^{* * \star K a g a w a}$ University, Hayashi-cho, Takamatsu, Kagawa, 761-0396, Japan \\ E-mail: nohmi@eng.kagawa-u.ac.jp

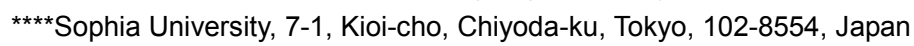 \\ E-mail: y-terumi@sophia.ac.jp
}

\begin{abstract}
In this paper, experiments on the motion of a tethered system with large deformation and large displacement are discussed. A tethered subsatellite in space environment is a well-known example of this system. However, even fundamental experiments on the motion of the tethered system have not been conducted. Also, from the viewpoint of flexible multibody dynamics, it is expected that simple experiments for the motion of a very flexible body should be completed first in order to verify the validity of the proposed approach. In this study, the tethered system consists of a very flexible body and a rigid body. First, we conducted an experiment on the fundamental motion of a very flexible body with an attached mass in gravity space. We selected steel and rubber as tether materials. In the experimental results, the characteristics of the complex motion of a very flexible body were obtained. Second, an experiment in microgravity space was done. The coupled motion between the very flexible body and rigid body was investigated. It was verified that the motion of the system with large deformation is excited in microgravity space, and there is the coupling motion between the deflection of the very flexible body and the rotation of the rigid body.
\end{abstract}

Key words: Multibody Dynamics, Experiment, Microgravity, Flexible Body, Large Deformation and Displacement, Rigid Body, Coupled Motion

\section{Introduction}

Space and sea could be useful environments for the human activity space in the future. It is necessary to develop a new mechanical system to use such an extreme environment. In such an environment, a tethered system that uses a cable or a wire rope for transportation has been considered ${ }^{(1)(2)}$. In general, a tether is a cable or a wire rope, and a tethered system consists of the tether and attached equipment. A cable and a wire rope have the advantage of light weight and compactable function. Furthermore this system doesn't cause environmental pollution because the system doesn't equip the thruster that needs fuel. Therefore a tethered system has various possibilities for engineering in extreme environmental conditions: for example, in space or deep in the sea. A tethered subsatellite in space is a well-known example of this system. It can be equipped with a camera and used

*Received 4 Dec., 2006 (No. T-04-1036) Japanese Original : Trans. Jpn. Soc. Mech. Eng., Vol.71, No.703, C (2005),

pp.821-828 (Received 17 Sep., 2004) [DOI: 10.1299/jee.2.64] 
to explore a structure when the mother ship is not able to approach this structure for maintenance $^{(3)-(6)}$. The tension of the tether has a large influence, even if the tension is small, because there is little influence of gravitational force acting in a constant direction in extreme environments such as space or deep in the sea. In such circumstances, the motion of the tether often has large deformation. Moreover, it is supposed that the motion of the tethered system is complex because the coupling motion between the tether and the attached equipment is excited. Therefore, it is important to observe the motion of the tethered system. However, it is difficult to perform experiments on the motion of a tethered system, and so experimental approaches on the motion of a tethered system have been almost nonexistent. Moreover, there have been no experiments of a tethered system in microgravity space. Also, from the viewpoint of flexible multibody dynamics, it is expected that simple experiments for the motion of a very flexible body should be completed first in order to verify the validity of the proposed approach ${ }^{(7)(8)}$.

In this paper, a system consisting of a very flexible body and a rigid body is regarded as the tethered system. A basic experiment of a tethered system was conducted for the following two purposes: first, to understand the motion of the tether and the coupled motion between a very flexible body and a rigid body; second, to obtain basic data to validate the proposed numerical approach for the motion of the tethered system. First, an experiment on the fundamental motion of a very flexible body with an attached mass is done under gravity. Two kinds of materials are used as tethers in this experiment. One is steel. The physical properties of steel are already known. In addition, a rubber material is used. Rubber has abundant axial expansion and contraction. Second, an experiment is done under microgravity. The tethered system using rubber as the tether material is selected from the results of the first experiment. This second experiment was conducted to examine the coupling motion between a very flexible body and a rigid body.

2. Experiment for tethered system motion with large rotation, deformation and translation under gravity

In this area, on the motion analysis of tethered systems, experimental examination of the motion with large deformation and displacement in a tethered system is almost non-existent. In this paper, the case in which the mother ship moves as shown in Fig. 1 is assumed.

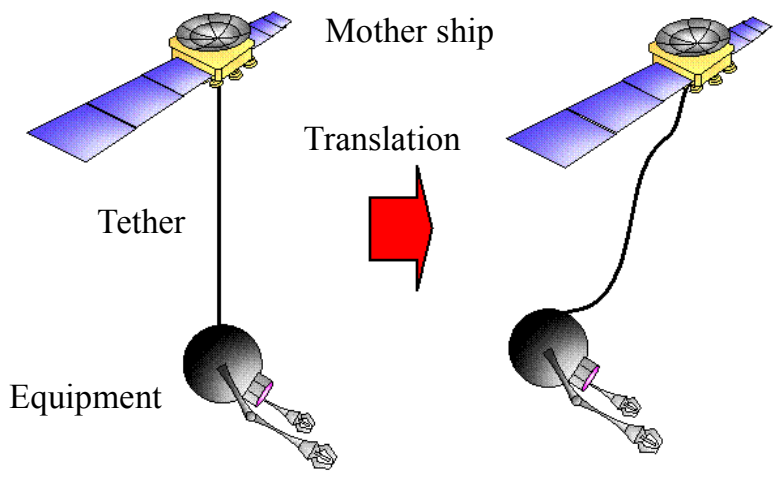

Fig. 1 The motion of a tethered system

\subsection{Motion of the bottom free tether using stainless steel material}

The purpose of this experiment is to investigate the motion of the tethered system with large deformation and displacement, using a stainless steel lamina as the model of the tether, and to obtain basic data to evaluate the validity of the numerical analysis. Even though this is a basic experiment, there are extremely few results available from other studies. This experimental result could possibly be used to validate the results of modeling and formulation in flexible multibody dynamics. 


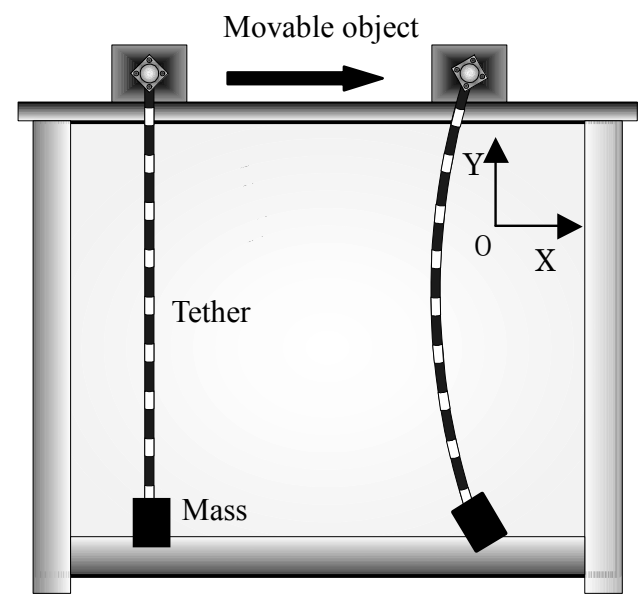

Fig. 2 Experimental Device

In this experiment, it is necessary to measure the tether motion by a no-contact method because the tether is light and the motion with large deformation and large displacement is excited. Therefore, the tether is measured by digital video cameras. The image data measured by the two digital video cameras is visualized using software. The software can determine three-dimensional location information of an arbitrary point on the object body based on the brightness information in the image data.

In the experimental device, the movement of one end of the tether is given by the movable object, as shown in Fig. 2. The top of the tether is fixed to the movable object with a rotation joint. The movable object controls the amount of straight advancement movement. A mass is attached at the bottom end of the tether. The joint between the movable object and the tether can rotate in two dimensions. Stainless steel SUS304 is used as the tether. This stainless steel lamina allows tether motion with large deformation. Reflection tape is pasted on the tether for measurement. The parameters of the tether material and the attached mass are as follows:

Tether length: 1.02 [ $\mathrm{m}]$, Density: $8030\left[\mathrm{~kg} / \mathrm{m}^{3}\right]$, Sectional area: $1.6 \times 10^{-6}\left[\mathrm{~m}^{2}\right]$, Thickness: $0.2 \times 10^{-3}[\mathrm{~m}]$, Width: $8.0 \times 10^{-3}[\mathrm{~m}]$, Young's modulus: $1.97 \times 10^{11}\left[\mathrm{~N} / \mathrm{m}^{2}\right]$, Mass at the bottom end: $4.0 \times 10^{-2}[\mathrm{~kg}]$.

In this case, the tether is very flexible though it is rigid for axial deformation. The movement of the upper end of the tether is shown in Fig. 3. The upper end of the tether is moved $0.55 \mathrm{~m}$ and its moving speed is $0.98 \mathrm{~m} / \mathrm{s}$. In the initial stage, the tether is in a static equilibrium state. The experimental result of the variation of the shapes of the tether on the

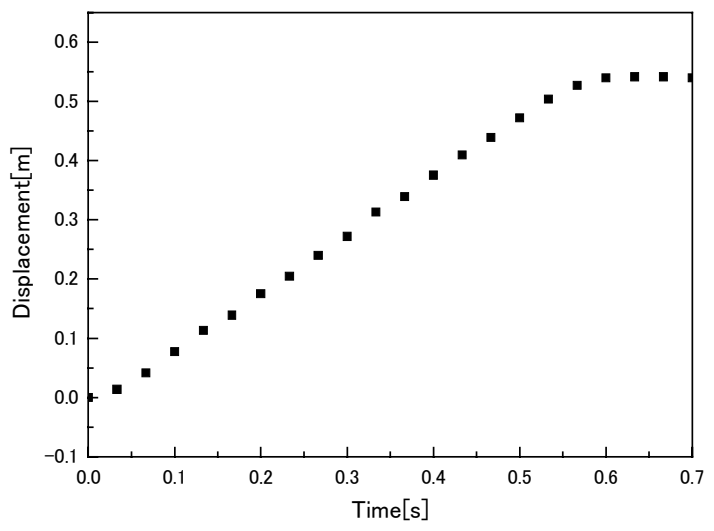

Fig. 3 Displacement at the upper end of the tether 

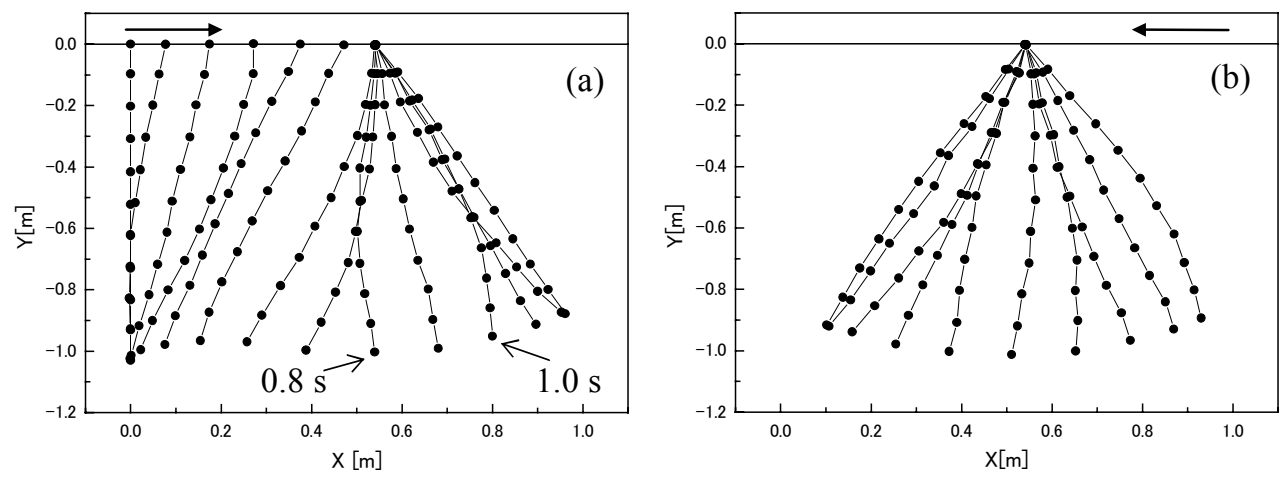

Fig. 4 Shape of the system using a stainless steel tether

(a) outward trip

(b) return trip

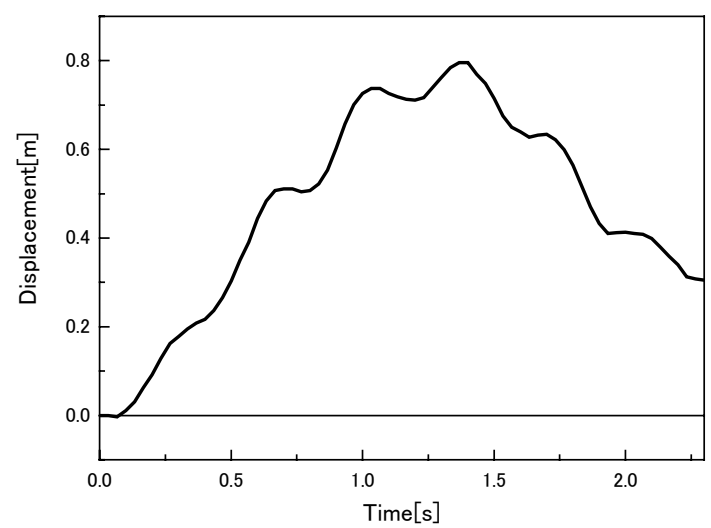

Fig. 5 Time history of midpoint of the tether using a stainless steel tether

outward trip and return trip are shown in Fig. 4. In this figure each black circle is an actual measurement point. The translation of the upper end causes lateral deformation of the tether. Then, the deformation increases with movement. The motion as the bottom mass is swung by the influence of the tether motion is observed. Its mode shape is like the first mode of a cantilever beam at $0 \mathrm{~s}$ to $0.7 \mathrm{~s}$. After this, the deflection of the middle point of the tether increases in the direction opposite to the traveling direction at $0.8 \mathrm{~s}$ to $1.0 \mathrm{~s}$. This phenomenon is caused by the coupling motion between the deflection of the tether and the inertia force of the mass. The mode shape at this time is not only like the first mode of a cantilever beam but also like the second mode of a cantilever beam. Large deformation by the inertia force of the bottom mass and the relation between the position of the upper end of the tether and the bottom mass is observed when the upper end of the tether stops. The time history of the lateral displacement of the midpoint of the tether during its travel is shown in Fig. 5. Then, tether motion with oscillation about the midpoint of the tether is observed. This phenomenon is caused by the ratio of the bottom mass and the mass of the tether. In this experiment, the ratio of the bottom mass and the mass of the tether is $3: 1$. The motion of the first mode and second mode together is excited because the tether motion is an influence on the motion of the system.

Therefore, it is confirmed that the complex motion is excited by the flexibility of the tether and the ratio of the bottom mass and the mass of the tether, even though the tethered system with large deformation and translation is a simple system consisting of a steel tether and a mass. 


\subsection{Motion of the bottom free tether using the rubber material}

By using the rubber material, remarkable axial expansion and contraction, nonlinear coupling of the axial and horizontal vibration and nonlinear elasticity are observed. A large deformation problem including these phenomena can be examined. In addition, rubber is useful because space is limited on a laboratory scale, and an actual tethered system is generally a large-scale system. In other words, measuring motion with large deformation is difficult because of the length limitation of the tether. With this limitation, the experiment using a tether with a low degree of elasticity is effective. In this experiment, first, the expansion to a static load is measured with a spring scale because the measurement of the dynamic physical properties of the material is difficult. Figure 6 shows the stress-strain curve. For this material, it is understood that Young's modulus decreases as the strain increases. The parameters of the tether material and the mass at the bottom are as follows:

Tether length: $1.0[\mathrm{~m}]$, Density: $0.014\left[\mathrm{~kg} / \mathrm{m}^{2}\right]$, Mass at the bottom: $3.0 \times 10^{-3}[\mathrm{~kg}]$, Sectional area of the tether: $7.069 \times 10^{-6}\left[\mathrm{~m}^{2}\right]$, Diameter of the tether: $3.0 \times 10^{-3}[\mathrm{~m}]$.

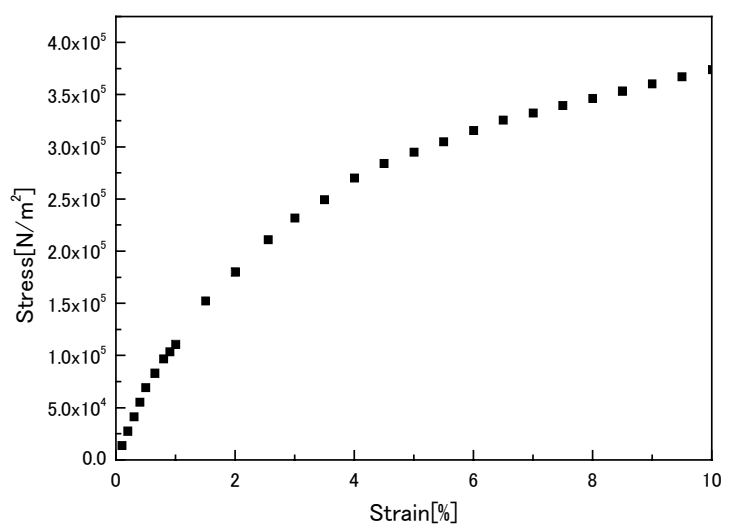

Fig. 6 Stress-Strain curve of the rubber material

Two kinds of models are used: one with the bottom mass and one without the bottom mass. The experimental device and the measuring method are the same as those used for the experiment using stainless steel. There are two purposes of this experiment. The first is to observe the coupling motion between the bottom mass and the tether, in which expansion and contraction are abundant. In this case, the ratio of the bottom mass and the mass of the tether is 1:3. Because the vibration phenomenon appears too strongly due to longitudinal elasticity, the motion of the tether deviates from the planar motion. The second purpose is to examine the influence of the mass, that is, the influence of tension, on the behavior of the tether with the mass compared to the tether without the mass. The compulsion movement of the tether end is given as well as the preceding clause. The experimental results of the variation of the shapes of the tether with the bottom mass in the outward and return trip are shown in Fig. 7. The experimental results of the variation of the shapes of the tether without the bottom mass in the outward and return trip are shown in Fig. 8.

First, the result of Fig. 7 is examined. The translation of the upper end causes the deformation of the tether. Then, the motion with large deformation is excited before the upper end of the tether stops. The difference of this result compared to the steel result is that the propagation speed of the transverse wave is very small, so the lateral oscillation is not great. Its mode shape is more complex than the shape of the steel tether. This phenomenon is caused by the boundary condition and the flexibility of the tether. This result is similar to the steel result of large deformation. However, a definite difference between the rubber result and the steel result is confirmed from the oscillation of the midpoint of the tether. The time history of the lateral displacement of the midpoint of the tether is shown in Fig. 9. The oscillation about the midpoint of the tether using the rubber tether is less clear than the steel 

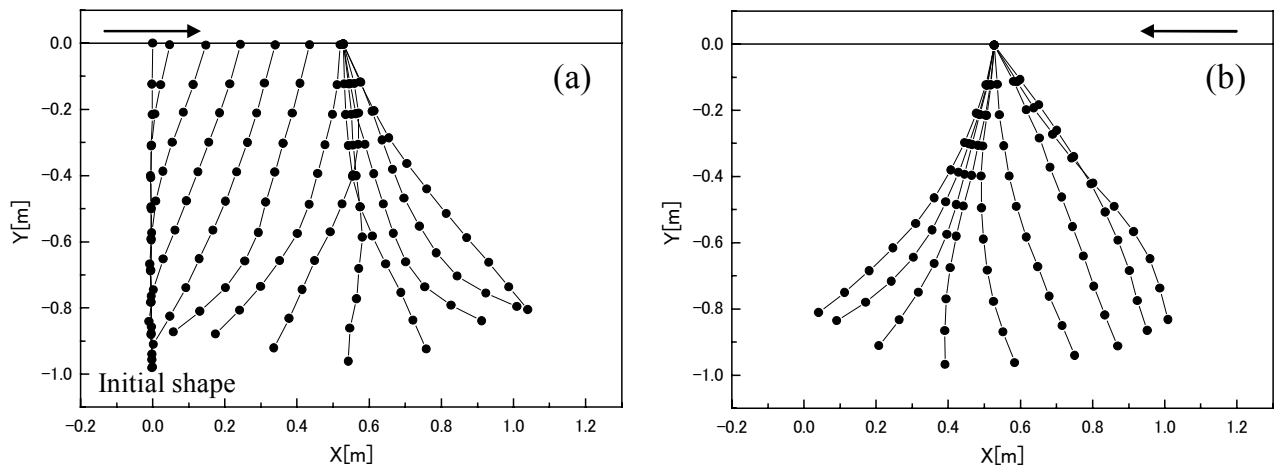

Fig. 7 Shape of the system using a rubber tether with the mass

(a) outward trip (b) return trip
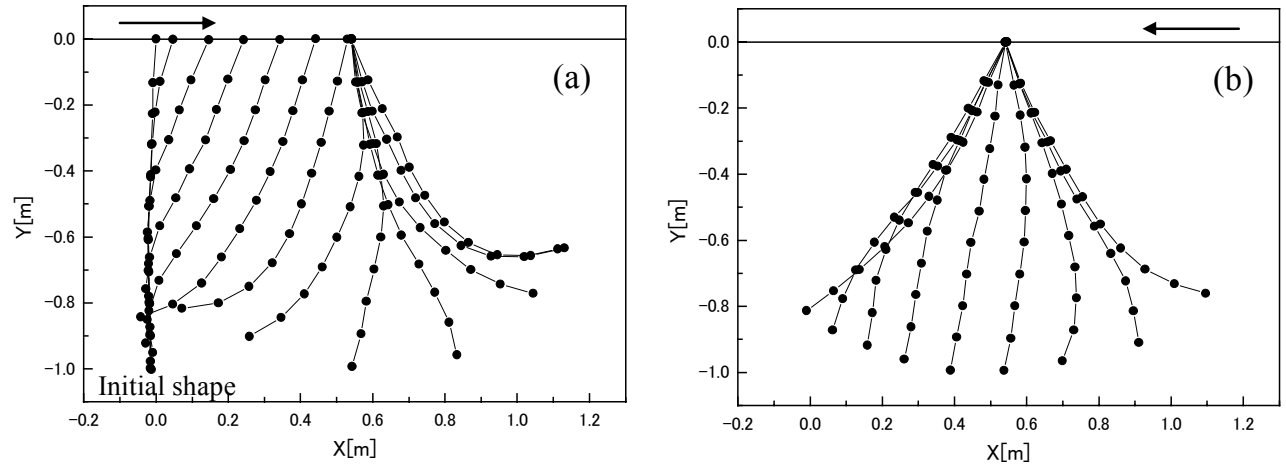

Fig. 8 Shape of the system using a rubber tether without the mass

(a) outward trip (b) return trip

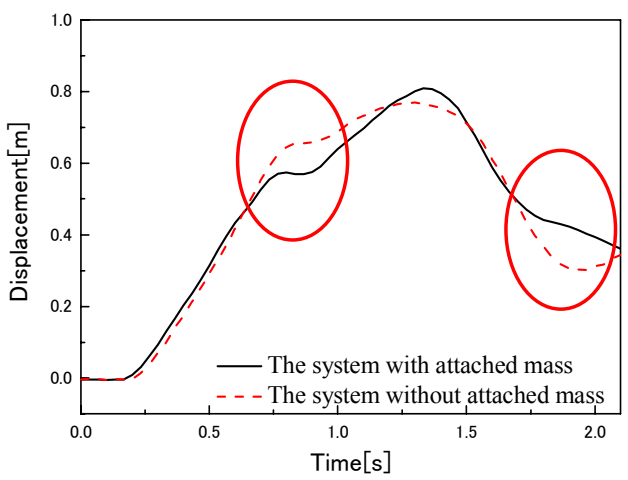

Fig. 9 Time history of midpoint of the rubber tether

result. This phenomenon occurs because the bottom mass is smaller than the mass of the tether and because the propagation speed of the transverse wave is very small for a tether with a low degree of elasticity. Therefore, a mode shape like the first mode of a cantilever beam becomes predominant. Next, the result of Fig. 8 is examined. The difference between the result of the model without the bottom mass, as compared to the model with the bottom mass, is obvious due to its jumping up behavior at the maximum displacement of the bottom of the tether. Jumping-up behavior is observed in the case of a pendulum with generally large amplitude. Because the inertia of the bottom mass provides the tension in the tether, and the deflection of the tether is small, this difference is caused. Therefore, the mass of the attached equipment has a very large influence on the motion of the tethered system with large deformation, even if the mass of the attached equipment is small. In other words, this result shows the motion of the tethered system is greatly changed by the motion 
of the attached equipment. In addition, the influence of the mass can be confirmed in the circle in Fig. 9. The system without the bottom mass has a larger amplitude. In other words, the deformation of the system without the bottom mass is larger. Therefore, the propagation of the transverse wave was confirmed by using the rubber tether without enlarging the length of the tether in this experiment. The rubber tether is thus suitable for discussing the coupling motion between the tether and rigid bodies. The rubber material is used as the tether in the following experiment under microgravity because of the findings obtained in this experiment under gravity.

3. Experiment for coupling motion between a very flexible tether and attached rigid body under microgravity

The purposes of this experiment are to observe the coupling motion between the tether and the rigid body and to obtain basic data to verify the numerical result of the formulation technique ${ }^{(7)(8)}$. The experiment under microgravity is selected because the tether moves with large deformation and the rigid body moves with large rotation, even if the tension is small. Therefore, the coupling motion between the tether and the rigid body is excited.

\subsection{Experimental method under microgravity}

A drop experiment facility provides a good microgravity environment, which is obtained by freely dropping a capsule in a vacuum. This microgravity environment continues for about $4.5 \mathrm{sec}$. Figure 10 shows the fall tower and fall capsule. The fall tower and fall capsule are composed of the following: a vacuum lock chamber, capsule separation device, capsule collection device, capsule support device, and a vacuum tube. The vacuum tube is set up under the soil and is composed of a free-fall part and a braking part. The tube is $150 \mathrm{~m}$ in total length; the free-fall part is $100 \mathrm{~m}$ and the braking part is $50 \mathrm{~m}$. The tethered system can fall in a steady state because the capsule falls in the vacuum. The initial state of the tethered system can be hardly changed and it will fall because the capsule is separated by turning off the power supply of the electromagnet that holds the capsule. It is possible for the capsule to brake by the cylindrical friction dump truck in $98 \mathrm{~m} / \mathrm{s}^{2}$ or less. For the collection of the capsule, the capsule in the braking part is held mechanically, and then the capsule is collected.

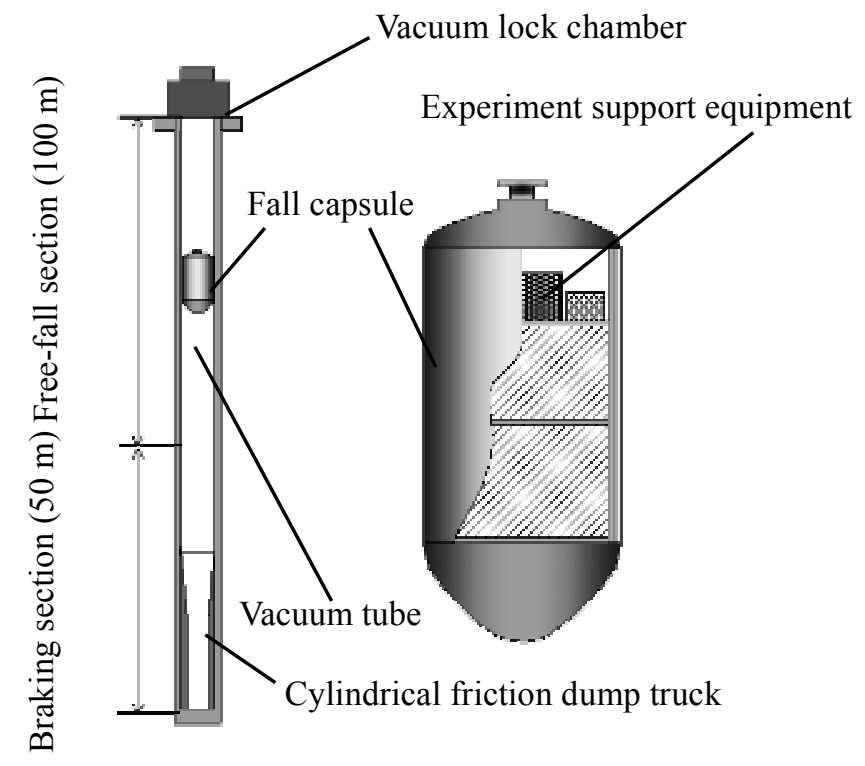
(a) Fall tower
(b) Fall capsule

Fig. 10 Experimental facility 


\subsection{Measuring method under microgravity}

To conduct this experiment, the measuring method is first selected, and then the size of the experimental device is decided. It is necessary to put the observation object as well as the measurement and data logging in the capsule because the space for the experiment is completely isolated and limited as shown in Fig. 10 (b). Wireless data is transmitted from the capsule become unstable while falling. The measures for impact are important in the case of repeated experiments because an acceleration of $98 \mathrm{~m} / \mathrm{s}^{2}$ acts on the capsule when braking. The measuring device adapted can observe the motion of the tethered system in the shaded part of the capsule. Figure 11 shows the entire measuring device. It is composed of two digital video cameras (DV), two CCD cameras, a diode luminescence circuit, lighting, a machine for the CCD camera (a wide-angle lens and a power supply adaptor are contained in the CCD) and the screen division machine. The measured object should always be in sight of the two cameras, and sufficient light to shine on the measuring point is needed. If the light of the lighting device overlap with the measuring point, the measuring point cannot be measured on the image. Moreover, contrast can be provided by background the lighting, as in a spotlight. Therefore, sufficient quantities of light are achieved by appropriately arranging and using two or more portable fluorescent lamps. The entire lighting device must be set up in limited space.

Some margins of error exist at the start time of each DV that records the image from the two CCD cameras, though it is necessary to measure the two images in the same period of time. The moment that the capsule enters the state of microgravity is distinguished by the projection of light emitted from the diode. This diode shines when detecting the microgravity signal from the capsule. The experimental device is arranged in the capsule, and the capsule is sealed up completely. Then the capsule is moved to the fall tube. In this time, there is a possibility that problems may occur because of the shaking in the capsule. Therefore, the state inside the capsule should be confirmed just before the capsule falls. Then, the images received from the several cameras inside the capsule can be put together on one screen using the screen division machine. The image in the capsule can be confirmed by receiving the image by wireless transmission on the ground side.

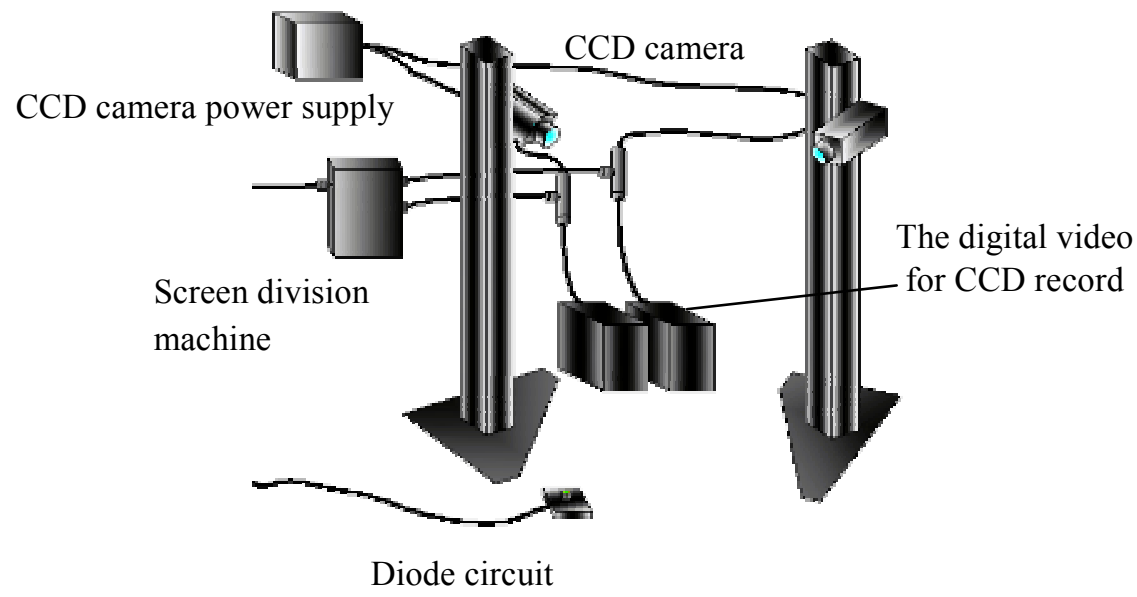

Fig. 11 Measuring device

\subsection{Experimental device under microgravity}

The experimental device can allow large deformation and large translation of the tethered system by recording the translation of the end plate moving at a constant speed. Figure 12(a) shows the experimental device. The experimental device consists of the moving end plate displacement device, the tethered system, and the rigid body support stand. The moving end plate displacement device consists of the DC motor, ball screw, and 
moving end plate. This device has the ability to perform the translation of the moving end plate by the DC motor that rotates the ball screw. The translation of the moving end plate is possible because the rotation of the moving end plate is controlled by the four surrounding L-type pillars. The reason for using this method is the moving end plate moving along the ball screw produces accurate translation. The DC motor starts when the microgravity signal is detected from the capsule. When the moving end plate approaches the ball screw edge, the limit switch is set up to stop automatically as a safety mechanism. The moving end plate displacement device was set to height $0.6 \mathrm{~m}$, width $0.15 \mathrm{~m}$, and depth $0.1 \mathrm{~m}$ because there was a limitation in the size of the capsule. The size of this device is assumed to be the maximum size to allow enough time for the tether motion with large displacement. Figure 12(b) shows the rigid body support stand. This device was made because the external force did not work under microgravity. The plinth rotates around according to the power of the spring, as shown in Fig. 12(b). Moreover, the stopper is installed because there was a danger of a fall from the support stand by the capsule shaking. Figure 12(c) shows the joint part of the tethered system. Two rigid bodies must be tied through the axis, so that they are attached at the rotation axis. The joint holes of the tether are passed through the axis of the rigid bodies. The tether and rigid bodies are united to become a free rotation in two dimensions. The tether was fixed to the moving end plate, and reflection tape was pasted on the tether for measuring.

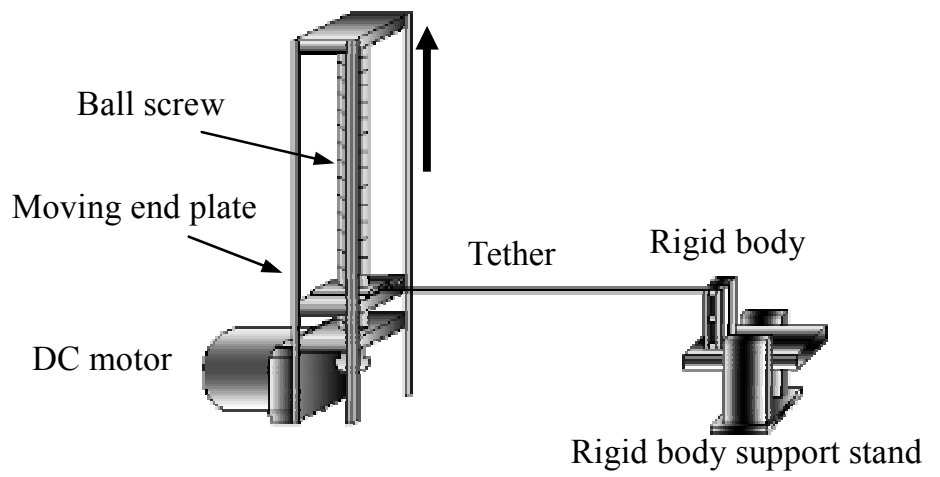

(a) Experimental device

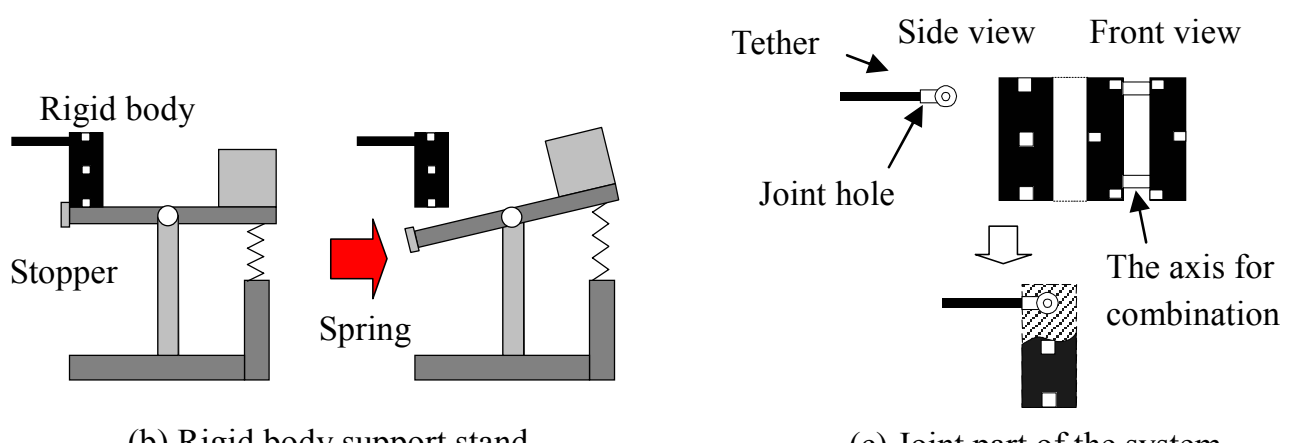

(b) Rigid body support stand

(c) Joint part of the system

Fig. 12 Experimental device

\subsection{Experimental results under microgravity}

Figure 13 shows the initial state of the tethered system. The parameters of the tether and the rigid body are tether length: $0.26[\mathrm{~m}]$, mass of the rigid body: $0.12[\mathrm{~kg}]$, rigid body length: $0.05[\mathrm{~m}]$, initial angle between the tether and the rigid body: $\pi / 2[\mathrm{rad}]$.

The time history of the vertical movement of the tether end is shown in Fig. 14. The tether moves with constant speed of $0.14[\mathrm{~m} / \mathrm{s}]$ except for a short period of acceleration. The time history of the variation of the acceleration of gravity in the experiment is also shown in 
Fig. 15. The experimental results of each shape of the tethered system in 0.2-s intervals are shown in Fig. 16. The end plate moves on the Y-axis in this figure. In the initial stage, the lateral deflection in the tether propagates. When the deflection of the tether reaches the end attached to the rigid body, the rigid body receives the moment due to the tension acting on the connecting point. Then the state of the motion of the tethered system is decided by the rotation of the rigid body and the deflection of the tether acting on each other. To explain this phenomenon, the angle of the rigid body and the amount of the deflection in the direction normal to the axis that connects both ends of the tether are considered, as shown in Fig. 17. The amount of the deformation of the tether can be discussed by considering this angle. The experimental results of the angle of the rigid body and the deflection at some positions in the tether are shown in Fig. 18(a) and (b). The experimental results of the total deflection at some positions in the tether are shown in Fig. 19 as one guide for evaluating the deformation of the tether. The five dotted lines in these figures show the time when the angle of the rigid body changes significantly. At these five points, the deflection of the tether becomes small. In other words, the tether has a straight shape and the tension acts on the connecting point so that the moment is given to the rigid body. Moreover, a decrease of the tether deflection is observed at the dotted lines in Fig. 19. This situation is thought to occur because the tether acts as a constraint due to the bend rigidity and the tension. Then this time corresponds to the time when the rotation of the rigid body changes. It is confirmed that the mechanism of the coupling motion exists. That is, the tension of the tether is excited because the deflection of the tether is decreased by the rotation of the rigid body. Then, the rotation of the rigid body is excited by this tension again.

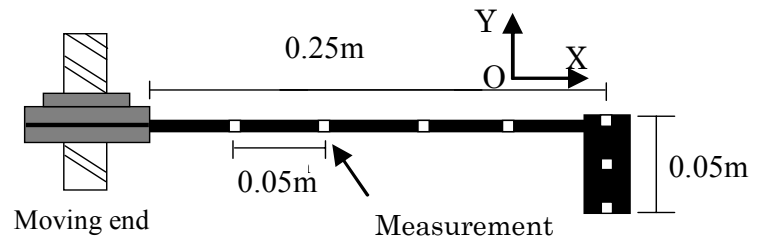

Fig. 13 Initial condition of the tethered system

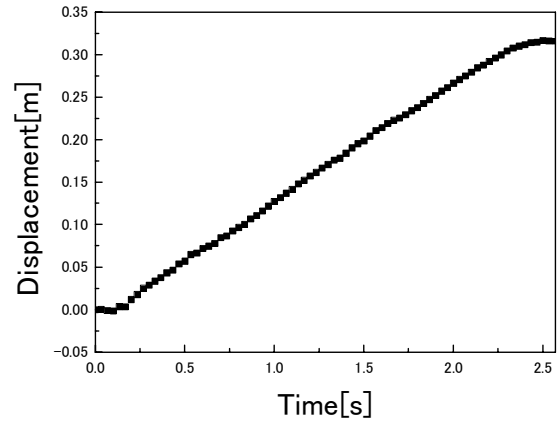

Fig. 14 Time history of moving end

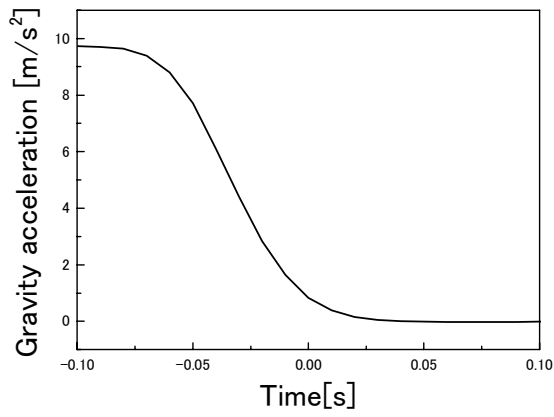

Fig. 15 Time history of gravity
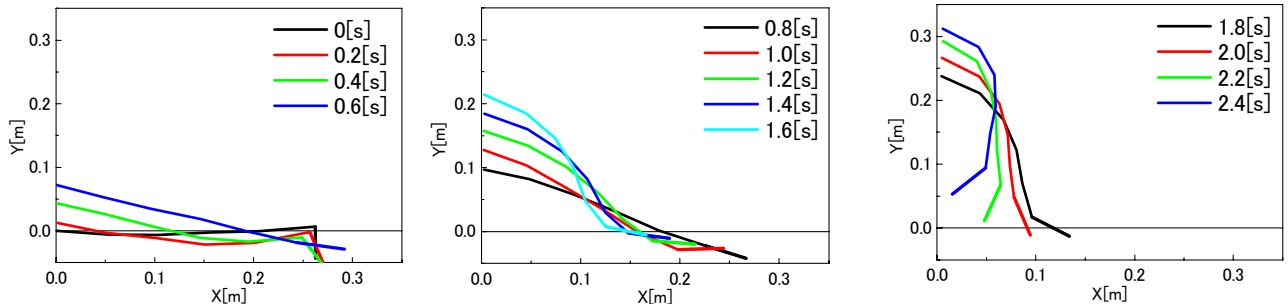

Fig. 16 Experimental results of the shape of the tethered system 


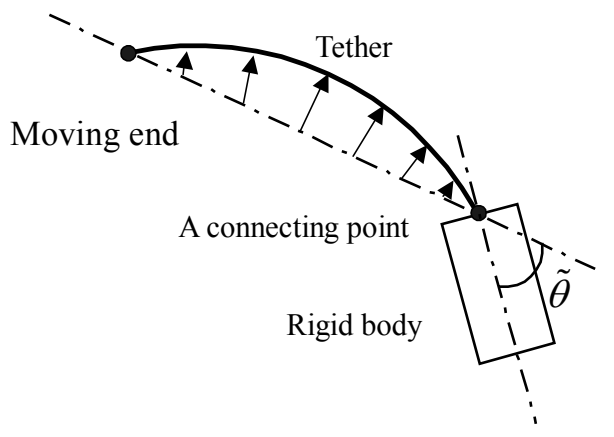

Fig. 17 Rotation angle of a rigid body

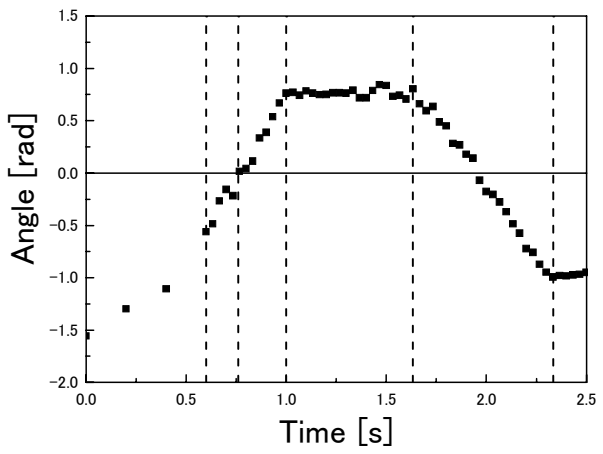

(a) Angle of rigid body

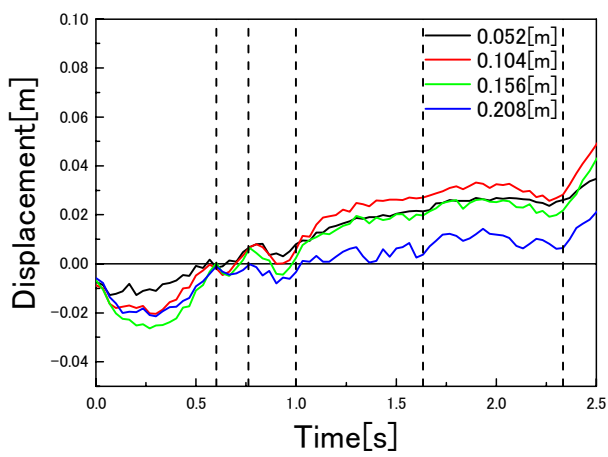

(b) Deflection of tether

Fig. 18 Time history of the tether motion

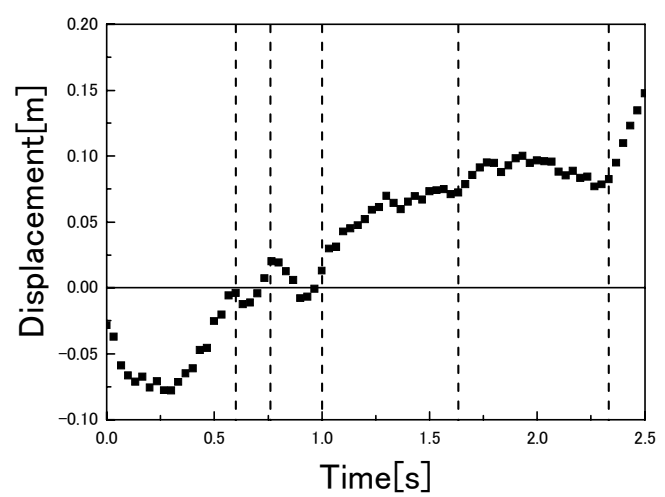

Fig. 19 Time history of overall tether translation

\section{Summary and conclusion}

In this paper, basic experiments of a tethered system were conducted to understand the motion of the tether and the coupled motion between a very flexible body and a rigid body. The experimental results using parameters in which the motion of the tether with large deformation and translation is remarkable are primarily shown here. These experimental results are made available in order to verify the validity of the numerical approach for the motion of a very flexible body.

First, an experiment of the bottom free tether was conducted. Stainless steel is used as a tether to obtain basic data. Rubber material that has the feature of remarkable axial expansion and contraction was used next. In the experiment result for the stainless steel tether, it was confirmed that the complex motion is excited by the flexibility of the tether 
and the ratio of the bottom mass and mass of the tether, even though the tethered system with large deformation and translation was a simple system consisting of a steel tether and a mass. In the experimental result for tether made of rubber material, the difference of this result compared to the steel result is that the propagation of the transverse wave is remarkable. It was confirmed that the influence of the boundary condition according to the quantity of the mass slightly changes the motion of the tether.

Second, an experiment on the system consisting of the tether and rigid body was conducted under microgravity. This experiment examined the coupling motion between a very flexible body and a rigid body. The experimental result shows that the large deformation of the tethered system is excited easily under microgravity. It was confirmed that the mechanism of coupling motion was present. That is, the tension of the tether is excited because the deflection of the tether decreases by the rotation of the rigid body. Then, the rotation of the rigid body is excited by this tension again.

\section{Acknowledgments}

This research was supported in part by the Japan Space Forum.

\section{References}

(1) Nohmi M., Nenchev D. N., Uchiyama M., Translation of a Tehtered Robot in Space by Casing Strategy, Transactions of the Japan Society of Mechanical Engineers, Series C, Vol. 64, No. 618 (1998), pp. 629-635.

(2) Nohmi M., Nenchev D. N., Uchiyama M., Motion Control of a Tethered Space Robot During Casting, Transactions of the Japan Society of Mechanical Engineers, Series C, Vol. 66, No. 647 (2000), pp. 2255-2261.

(3) Misra A.K., Dynamics and Control of Tether Connected Two-Body Systems, Proceedings of the 33rd Congress of the International Astronautical Federation (1982), p. 82.

(4) Koyama K.,Shuttle/Tethered Satelite System (TSS) andits Application to Space Science, Bulletin of the Institute of Space and Aeronautical Science, University of Tokyo(ISSN 05638100), 16-2(A), (1980), pp. 935-952.

(5) Nohmi M., Translation and End-effector Position / Attitude Control of a Tethered Space Robot, Doctor Thesis, Tohoku University, November, 1998.

(6) Marine survey frontier, Japan Society for Marine Surveys and Technology, (1993), pp. 201-239.

(7) Takehara S., Terumichi Y., Nohmi M., Sogabe K., Motion of a System Consisting of a String and Rigid Bodies at Its End, Transactions of the Japan Society of Mechanical Engineers, Series C, Vol. 69, No. 678 (2003), pp. 349-355.

(8) Takehara S., Terumichi Y., Nohmi M., Sogabe K., Dynamic Response of a Tethered System with Large Deformation and Large Displacement under Attitude Control, Transactions of the Japan Society of Mechanical Engineers, Series C, Vol. 70, No. 689 (2004), pp.105-112. 